\title{
X-ray induced photocurrent characteristics of CVD diamond detectors with different carbon electrodes
}

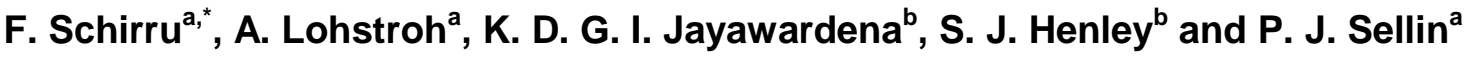 \\ ${ }^{a}$ Department of Physics, University of Surrey, \\ Guildford, GU2 7XH, United Kingdom \\ ${ }^{b}$ Advanced Technology Institute, University of Surrey, \\ Guildford, GU2 7XH, United Kingdom \\ E-mail: f.schirruegsi.de
}

\begin{abstract}
Diamond has unique properties which make it suitable for a broad range of radiation detection applications ranging from particle timing and spectroscopy, to neutron, UV and $\mathrm{X}$-ray sensors. In $\mathrm{X}$-ray dosimetry, the atomic number of diamond $(\mathrm{Z}=6)$ close to that of the human tissues $(Z=7.42)$ allows to mimic the real absorbed dose avoiding off-line recalculations. Moreover, its low atomic number and the capability to withstand high radiation fluxes make possible its use as beam monitor without altering significantly the properties of the interacting beam.

To preserve the tissue equivalence of the diamond and minimize the perturbation and absorption of the incident beam, diamond detectors based on low thickness and low atomic number electrodes become a requirement.

In this paper we present the X-ray detection characteristics of electronic grade CVD diamond sensors prepared in house with thin amorphous carbon electrodes fabricated by Pulsed Laser Deposition (PLD) technique in the fluence range of $2.3-3.6 \mathrm{~J} \cdot \mathrm{cm}^{-2}$. The devices showed a linear dependence of the induced photocurrent respect to the dose rate. Also, best dynamic response and better stability of the signals were achieved for applied bias up to $\pm 50 \mathrm{~V}$ with signal to noise ratio (SNR) of $\sim 300$.
\end{abstract}

KEYWORDS: Diamond detectors; Dosimetry concepts and apparatus; X-ray detectors.

\footnotetext{
* Corresponding author.
} 


\section{Contents}

1. Introduction

2. Material and methods $\quad 1$

3. Results and discussion 3

4. Conclusions 6

\section{Introduction}

High quality synthetic diamonds are of interest in a broad range of radiation detection applications ranging from particle timing and spectroscopy, to neutron, UV and X-ray sensors. Due to its near tissue equivalence [1], diamond is of great interest for medical dosimetry while its capability to operate at high temperature [2] along with the high thermal conductivity [3] and the low atomic number make it an attractive material for high intensity X-ray and particle beam monitoring. In these applications however it becomes an absolute requirement to use diamond detectors with electric contacts having low thickness and low atomic number. In fact, electrodes with such characteristics help to minimize the perturbation and absorption of the incident beam and preserve the tissue equivalence of the diamond material.

The Pulsed Laser Deposition (PLD) technique can be used to produce metal-free amorphous carbon coatings as electrode material for diamond detectors, as already reported in [4]. PLD offers many advantages among which: i) compared to other conventional techniques such as thermal evaporation, deposited ions and atoms impinge onto the substrate surface with high energy (>100 eV) [5] giving better adhesion and mechanical stability of the layer and ii) by changing the deposition parameters such as laser power and ambient atmosphere [6] it allows to create ad-hoc amorphous carbon layers having a determined structure in terms of $\mathrm{sp}^{2} / \mathrm{sp}^{3}$ bonding ratio, conduction mechanism and electrical/optical properties. In vacuum and at high laser fluence $\left(\sim 10 \mathrm{~J} \cdot \mathrm{cm}^{-2}\right)$ such method allows for example to produce smooth so-called Diamond-like Carbon (DLC) films with up to $90 \%$ of carbon atoms being sp $^{3}$ bonded.

This paper reports a preliminary study of the photocurrent characteristics under X-ray beam of radiation detectors based on electronic grade CVD synthetic diamond samples with amorphous carbon electrodes fabricated by PLD technique at different laser fluences. The performance of the devices was assessed by studying their leakage current, the dynamic response under irradiation, the stability and repeatability of the signal and their linearity with respect the dose rate. While in the paper [4] the authors investigated the dosimetric properties of a diamond sensor based on carbon electrodes fabricated at fixed laser fluence, in this work the PLD method is used on synthetic diamonds for a fluence dependent study.

\section{Material and methods}

The material considered in this work consists mainly of one electronic grade polycrystalline CVD (pcCVD) synthetic diamond sample purchased from Element Six Ltd whose dimensions are $10.0 \mathrm{~mm} \mathrm{x}$ $10.0 \mathrm{~mm} \times 0.3 \mathrm{~mm}$ with polished surfaces. Prior deposition of the electrodes, the sample was thoroughly cleaned in acetone, isopropanol and then washed in de-ionized water. Next it was mounted in a shadow mask in order to fabricate four circular electrodes of $3 \mathrm{~mm}$ in diameter on each of its sides. One side of the sample was covered with four $\mathrm{Al}$ contacts $70 \mathrm{~nm}$ thick followed by $30 \mathrm{~nm}$ of Au to prevent oxidisation of the Al layer. Deposition was performed by an Edwards K575XD Turbo Pumped High Resolution Sputter Coater. 

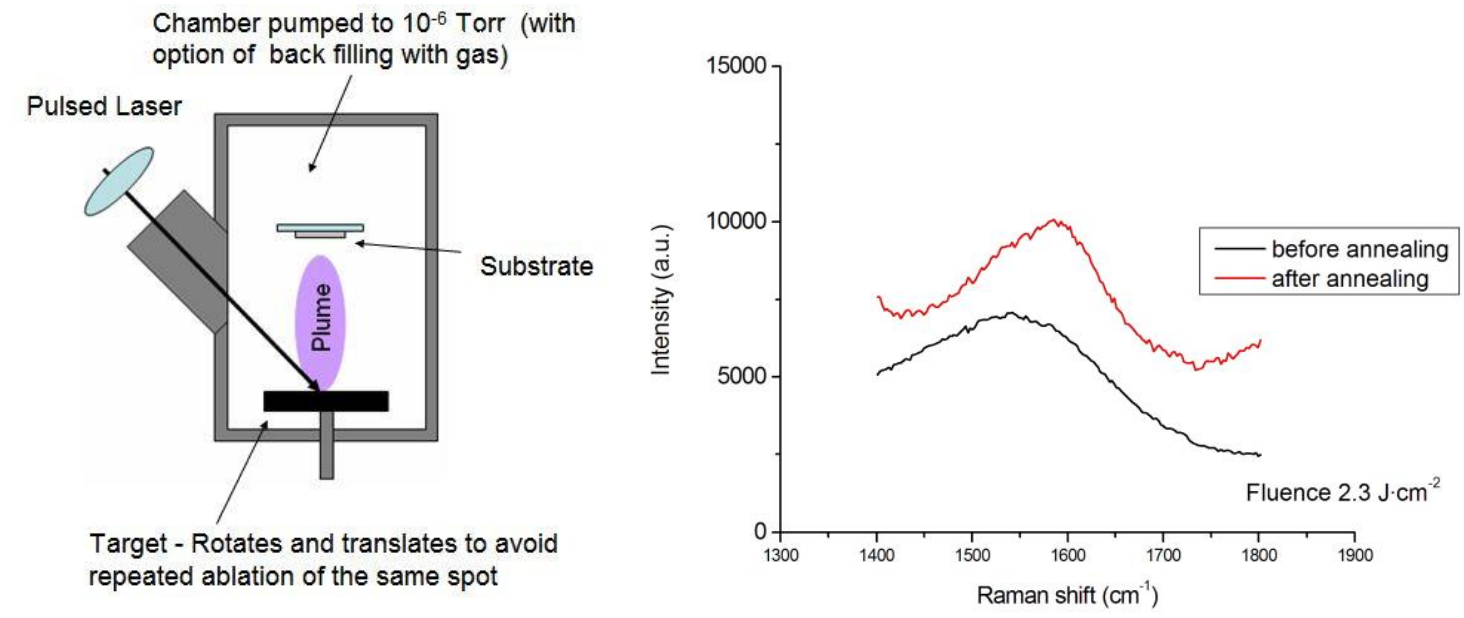

Figure 1. (Left) Schematic of the PLD system used to deposit the amorphous carbon electrodes. The laser fluences considered during the process of deposition were $2.3,2.8,3.2$ and $3.6 \mathrm{~J} \cdot \mathrm{cm}^{-2}$. (Right) Raman spectroscopy performed on the carbon electrode fabricated at the laser fluence of $2.3 \mathrm{~J} \cdot \mathrm{cm}^{-2}$. A positive shift of the $\mathrm{G}$ peak was observed after annealing the diamond sample.

Figure 1 (Left) reports a schematic of the PLD system used to fabricate the amorphous carbon electrodes. A graphite target is installed $6 \mathrm{~cm}$ apart from the diamond sample in a vacuum chamber kept at the working pressure of $10^{-6}$ Torr. A $25 \mathrm{~ns}$ pulsed $\mathrm{KrF}$ excimer laser (Lambda - Physik LPX 210i) operating at $248 \mathrm{~nm}$ is focused through a quartz window onto the graphite target which rotates at a speed of $40 \mathrm{rpm}$. The laser interacts with the target creating a plasma plume rich of highly energetic ions and carbon clusters which then deposit on the surface of the sample leading to the formation of an amorphous film that is more diamond-like in nature [7].

Four different amorphous carbon electrodes $20 \mathrm{~nm}$ in thickness were deposited on the second side of the sample by PLD method at the laser fluence of $2.3,2.8,3.2$ and $3.6 \mathrm{~J} \cdot \mathrm{cm}^{-2}$. The repetition rate of the laser pulses was $10 \mathrm{~Hz}$. It should be noted that during the fabrication process of the carbon layers, it was observed that higher values of thickness and laser fluence led to an unsustainable increase of stress of the deposited carbon film and consequently poor adhesion on the diamond surface.

The sample was next annealed at $500{ }^{0} \mathrm{C}$ for $5 \mathrm{~min}$ at the pressure of $10^{-2}$ Torr. The annealing was performed in order to improve both the adhesion of the carbon electrodes on the diamond surface and the electron delocalization through graphitization which leads to a better conductivity [8]. The sample was then installed onto a printed circuit board (PCB) connecting the top and back electrodes to the PCB signal lines through gold wires of $24 \mu \mathrm{m}$ in diameter.

The structure of the carbon coatings was assessed before and after annealing by Raman spectroscopy using a Renishaw MicroRaman system at the wavelength of $514.5 \mathrm{~nm}$. As reported in figure 1 (Right), the amorphous carbon layer produced at the laser fluence of 2.3 $\mathrm{J} \cdot \mathrm{cm}^{-2}$ shows a $\mathrm{G}$ peak centred at $\sim 1541 \mathrm{~cm}^{-1}$ which is linked to the presence of $\mathrm{sp}^{2}$ bonded atoms in the film. The peak shape is also rather consistent with the deposition of high $\mathrm{sp}^{3} \mathrm{DLC}$ [8]. After annealing the contact, a positive shift of the $G$ peak is observed which might suggest an increase in the graphitic phase of the amorphous carbon layer. Raman spectroscopy performed on the other carbon electrodes led to similar observations. For the fluence values reported in this work, the $\mathrm{sp}^{3}$ fraction for the pure carbon film is expected to be around 70\% [6]. 

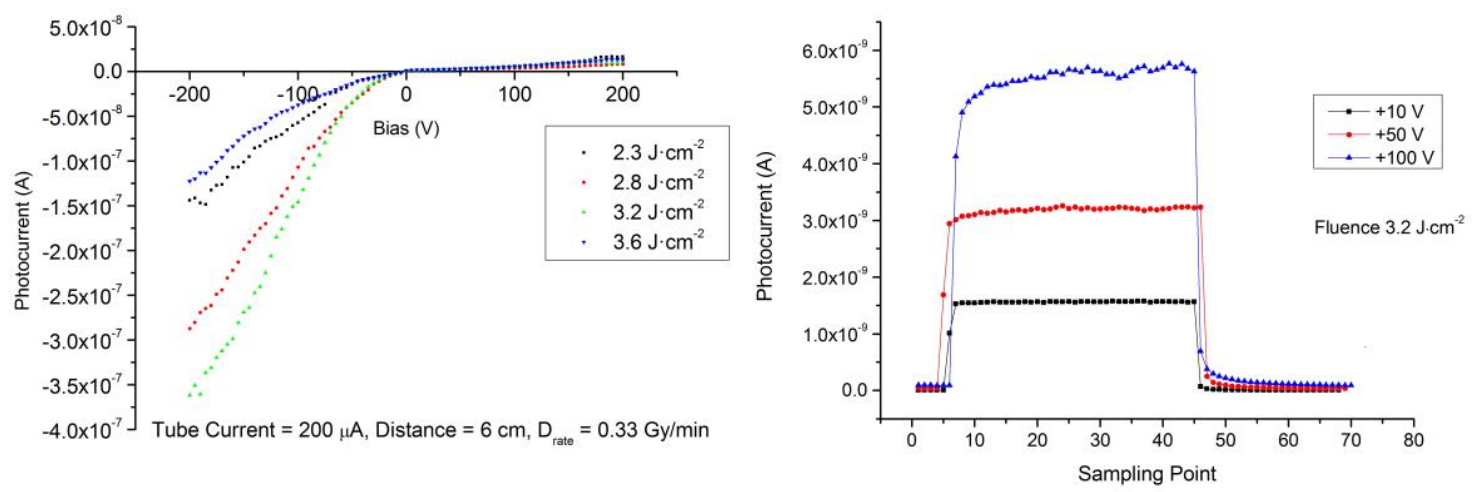

Figure 2. (Left) IV characteristics of the diamond devices recorded under X-ray beam at the dose rate of 0.33 Gy/min. (Right) Time evolution of the photocurrent recorded for the diamond sensor with carbon layer fabricated at the laser fluence of $3.2 \mathrm{~J} \cdot \mathrm{cm}^{-2}$. Best dynamic response is achieved applying a bias voltage of $+10 \mathrm{~V}$.

Photocurrent characteristics and dosimetric properties of the diamond detectors were investigated by using a $50 \mathrm{kV}_{\mathrm{p}} \mathrm{X}$-ray tube with a molybdenum reflection target (Mod. Oxford instruments XF50 11). Each front carbon electrode was carefully centred and placed at $6 \mathrm{~cm}$ from the X-ray source. At this distance, setting the tube current to $200 \mu \mathrm{A}$, the corresponding dose rate was $0.33 \mathrm{~Gy} / \mathrm{min}$ as assessed by a $0.6 \mathrm{~cm}^{3}$ ionization chamber Mod. NE2571A. Measurements of photocurrent, always under dark conditions, were performed with a Keithley 487 Pico Ammeter which served also as a voltage source to apply the appropriate bias voltage. The dosimetric characterization that follows has to be considered as a preliminary test since it spans over a narrow range of energies and dose rates.

\section{Results and discussion}

At first, the leakage current of each diamond device was tested as a function of the applied voltage in the range $\pm 200 \mathrm{~V}$ in $5 \mathrm{~V}$ steps. At $\pm 100 \mathrm{~V}$ the leakage current did not exceed $5 \mathrm{pA}$ leading to a minimum bulk resistivity of at least $2.8 \cdot 10^{14} \Omega \cdot \mathrm{cm}$. This value is in agreement with others reported in literature for polycrystalline diamond samples [9].

Figure 2 (Left) shows the photocurrent-voltage characteristics of the diamond detectors recorded under X-ray irradiation in the range $\pm 200 \mathrm{~V}$. The data points were recorded in steps of $5 \mathrm{~V}$ and calculated by averaging the photocurrent during a period of 30 seconds. According to the bias polarity applied, the induced photocurrent presented an asymmetric behaviour which became quasi-ohmic only for negative polarity. For voltages greater than $\pm 100 \mathrm{~V}$, all the devices reported instability of the photocurrent.

Figure 2 (Right) reports the photocurrent recorded for the diamond device with carbon electrode prepared at the laser fluence of $3.2 \mathrm{~J} \cdot \mathrm{cm}^{-2}$. Best dynamic response of the detector in terms of rise time, fall-off time and stability of the photocurrent under irradiation was achieved when operating at $+10 \mathrm{~V}$. As shown in the graph, higher values of voltage led to a slower response of the device after switching on the X-ray beam and bigger instability of the photocurrent during the irradiation.

Figure 3 (Left) shows the signal repeatability of the devices at $+10 \mathrm{~V}$ after a priming dose of 1 Gy. Each cycle consists of 2 minutes of irradiation followed by a one minute break. The diamond detectors show similar behaviour in terms of stability, dynamic response and magnitude of the recorded photocurrent. 

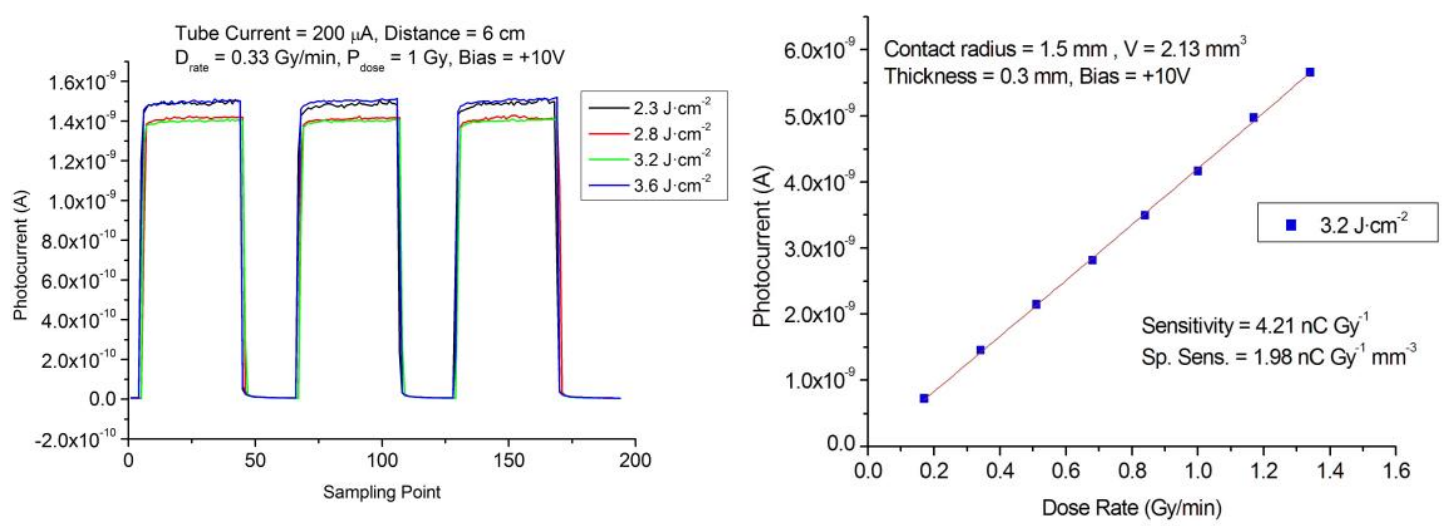

Figure 3. (Left) Signal repeatability of the diamond detectors. The photocurrent was recorded at $+10 \mathrm{~V}$ after a priming dose of $1 \mathrm{~Gy}$. The coefficient of repeatability was found to be within the $0.5 \%$ for all the devices. (Right) Dose rate linearity of the diamond detector with carbon electrode prepared at the laser fluence of $3.2 \mathrm{~J} \cdot \mathrm{cm}^{-2}$. The fit performed on the data points by using the Eq. (1) returned a parameter of linearity $\Delta=0.99$. The calculated sensitivity and specific sensitivity (per unit volume) of the device were $4.21 \mathrm{nC} \cdot \mathrm{Gy}^{-1}$ and $1.98 \mathrm{nC} \cdot \mathrm{Gy}^{-1}$ respectively.

Differences in the measured photocurrent are below the variations observed due to inaccuracy of alignment of the devices with respect the X-ray source, which, as assessed in a preliminary test, were estimated to be in the order of $10 \%$. In all cases a signal to noise ratio of $\sim 300$ was found assuming a value of leakage current, recorded between irradiations, of $5 \mathrm{pA}$. Such ratio becomes $\sim 900$ considering irradiations performed at a dose rate of $1 \mathrm{~Gy} / \mathrm{min}$.

The reproducibility of the signals was calculated by taking the percentage ratio of the standard deviation of the average photocurrent value under exposure of a fixed dose rate. In all cases, the obtained values are within $0.5 \%$ as recommended by the International Atomic Energy Agency (IAEA) [10] which indicates low fluctuations in the signals. From the data reported in figure 3 (Left) it was possible also to estimate the photocurrent rise and fall-off time of the devices, converted in time, in $\sim 2 \mathrm{~s}$. Such value could be of interest in IMRT dosimetry applications where fast response of the dosimeter is required in order to follow the fast transients of IMRT fields.

Figure 3 (Right) shows the dose rate dependence of the diamond detector with carbon electrode deposited at the laser fluence of $3.2 \mathrm{~J} \cdot \mathrm{cm}^{-2}$. Measurements were performed at the same conditions as described before by changing the tube current from $100 \mu \mathrm{A}$ to $800 \mu \mathrm{A}$ corresponding to dose rate values between 0.17 and $1.34 \mathrm{~Gy} / \mathrm{min}$.

Fowler model [11] predicts a dose rate dependence $D$ of the detector current $I$ as:

$$
I=I_{\text {dark }}+R D^{\Delta}
$$

where $R$ is a constant, $I_{\text {dark }}$ is the dark current and $\Delta$ an exponential parameter which describes the deviation from the linearity. It depends on both the material characteristics and experimental conditions such as applied electric field and flux of the beam. For all the devices under study $\Delta$ was found to be in the range $0.97-0.99$ which, according to the model, suggests a uniform trap distribution through the diamond bulk. This values are in agreement with those reported in literature $(0.86-1.035)$ for both PTW natural single crystal diamonds and CVD synthetic diamond devices $[12,13]$. The detector sensitivities were found between 4.03 and $4.28 \mathrm{nC} / \mathrm{Gy}$ which are comparable with the values calculated in [14] for polycrystalline diamond detectors. 

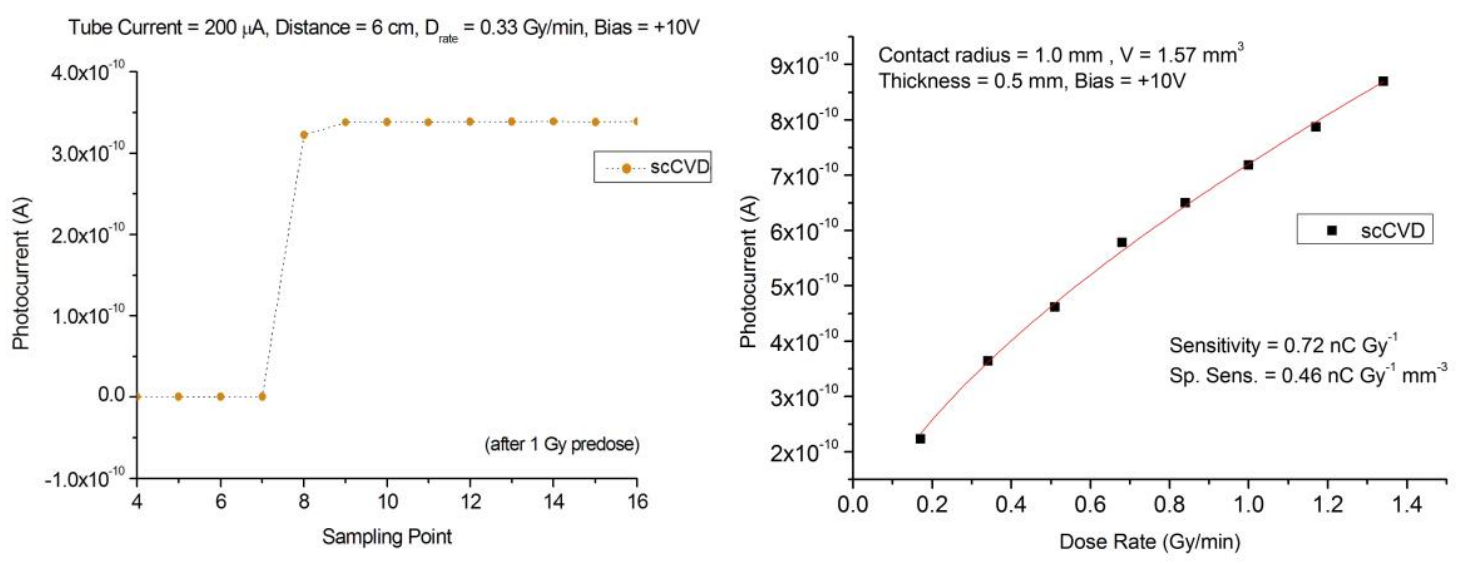

Figure 4. (Left) Photocurrent response of the scCVD diamond detector after switching on the X-ray beam. The device shows a rise time, converted in time of $\sim 1 \mathrm{~s}$. (Right) Dose rate dependence of the diamond detetector whose fit performed by Eq. (1) returned a parameter of linearity $\Delta=0.63$.

One of the quality parameters of a detector can be estimated by the knowledge of its gain factor $G$ defined as $I_{m} / I_{g}$ where $I_{m}$ is detector current measured and $I_{g}$ the current induced by the radiation field. The latter can be defined as:

$$
I_{g}=D \rho e v / w .
$$

For the case of the device fabricated with carbon electrode at the laser fluence of $3.2 \mathrm{~J} \cdot \mathrm{cm}^{-2}$ and assuming a dose rate $D$ of $0.33 \mathrm{~Gy} / \mathrm{min}$, diamond density of $\rho=3.5 \mathrm{~g} / \mathrm{cm}^{3}$, the sensitive volume of $v=2.13 \cdot 10^{-3} \mathrm{~cm}^{3}, e=1.6 \cdot 10^{-19} \mathrm{C}$ as electronic charge, $w=13 \mathrm{eV}$ as energy required to produce an electron-hole pair in diamond, we obtain a gain factor $G=0.28$. Such value is smaller respect to those reported in literature for commercially available natural diamonds which show a gain factor around $0.5[1,15,16]$. However, it should be pointed out that the gain factor is not only affected by bulk properties of the diamond sample but also by the experimental parameters such as the magnitude of the electric field applied on the device.

So far, it have been investigated the dosimetric properties of diamond detectors based on one pcCVD sample prepared with amorphous carbon electrodes in one side and $\mathrm{Al} / \mathrm{Au}$ metallic contacts in the opposite side. In a complementary study, another diamond device, based on a single crystal CVD (scCVD) sample of dimensions $4.0 \mathrm{~mm}$ x $4.0 \mathrm{~mm} \times 0.5 \mathrm{~mm}$, was fabricated by depositing on both sides $2 \mathrm{~mm}$ in diameter and $20 \mathrm{~nm}$ thick amorphous carbon electrodes at the laser fluence of $3.2 \mathrm{~J} \cdot \mathrm{cm}^{-2}$. After deposition of the electrodes, the sample was next annealed for the reasons and at the conditions previously described. Also in this case, the photocurrent characterization was performed by carefully centring and placing the sample at $6 \mathrm{~cm}$ from the X-ray source.

Figure 4 (Left) shows the rise time of the detector photocurrent recorded after delivering a priming dose to the device of $1 \mathrm{~Gy}$. The device showed stability of the current and fast dynamic response $(\sim 1 \mathrm{~s})$ under irradiation similar to those reported previously on polycrystalline diamond sensors. Applying a voltage greater than $+25 \mathrm{~V}$ the photocurrent showed strong instability preventing the use of the device itself. For this reason it was decided to perform all measurements applying a bias of $+10 \mathrm{~V}$. The leakage current before irradiation was $1.50 \cdot 10^{-13} \mathrm{~A}$ while the induced photocurrent under X-ray beam was $3.41 \cdot 10^{-10} \mathrm{~A}$. These values led to a signal to noise ratio of $\sim 2200$. 


\begin{tabular}{lccccccccc}
\hline Device & $\begin{array}{c}\text { Fluence } \\
\left(\mathrm{J} \cdot \mathrm{cm}^{-2}\right)\end{array}$ & $\begin{array}{c}\mathrm{I}_{\text {meas }} \\
(\mathrm{A})\end{array}$ & $\begin{array}{c}\mathrm{I}_{\text {dark }} \\
(\mathrm{A})\end{array}$ & $\begin{array}{c}\mathrm{I}_{\text {gen }} \\
(\mathrm{A})\end{array}$ & S/N & Gain & $\begin{array}{c}\mathrm{RT} \\
(\mathrm{s})\end{array}$ & $\begin{array}{c}\text { Sens. } \\
(\mathrm{nC} / \mathrm{Gy})\end{array}$ & $\Delta$ \\
\hline $\mathrm{pcCVD} 1$ & 2.3 & $1.49 \cdot 10^{-9}$ & $5.37 \cdot 10^{-12}$ & $5 \cdot 10^{-9}$ & 277 & 0.30 & 2 & 4.24 & 0.98 \\
& & & & & & & & & \\
$\mathrm{pcCVD} 3$ & 3.2 & $1.40 \cdot 10^{-9}$ & $4.82 \cdot 10^{-12}$ & $5 \cdot 10^{-9}$ & 290 & 0.28 & 2 & 4.21 & 0.99 \\
& & & & & & & & & \\
$\mathrm{scCVD}$ & 3.2 & $3.41 \cdot 10^{-10}$ & $1.50 \cdot 10^{-13}$ & $2 \cdot 10^{-9}$ & 2200 & 0.17 & 1 & 0.81 & 0.63 \\
\hline
\end{tabular}

Table 1. Dosimetric and electrical properties of the investigated diamond devices. Only two devices fabricated with the polycrystalline sample are shown. All the measurements were recorded biasing the diamond devices at $+10 \mathrm{~V}$.

Table 1 reports the main parameters calculated at $+10 \mathrm{~V}$ of some of the diamond detectors considered in this work. Surprisingly, the gain factor estimated for the device based on the scCVD diamond is lower than that obtained for the other devices based on the pcCVD sample. This is a direct consequence of the lower current recorded under irradiation. Such result could be related either to the smaller sensitive volume of the device and/or to the smaller magnitude of the electric field applied being, in this case, the thickness of the sample bigger. Another aspect to be considered is the exponential parameter $\Delta$ that for the scCVD sample was found to be equal to 0.63 as calculated by fitting the data points shown in figure 4 (Right). Such value denotes a sublinear relationship between the induced photocurrent and the dose rate. This result contradicts what is reported in [17] where a $\Delta$ close to one was found after annealing the device. However, it should be kept in consideration that the Fowler model assumes that $\Delta$ is determined only by the bulk properties of the material. In this case $\Delta$ could be also affected by the electrode interface [17]. Increasing the applied bias to $+25 \mathrm{~V}$ it was found $\Delta=0.86$. Such result denotes a dependence of $\Delta$ on the electric field applied on the device, as already reported by [18].

\section{Conclusions}

The aim of this study was to investigate the X-ray induced photocurrent characteristics of four radiation detectors based on one $300 \mu \mathrm{m}$ thick pcCVD diamond. Different amorphous carbon electrodes were deposited by PLD method at the laser fluence of 2.3, 2.8, 3.2 and 3.6 $\mathrm{J} \cdot \mathrm{cm}^{-2}$. However, the Raman spectroscopy performed on the carbon films revealed similarities in terms of the $\mathrm{sp}^{2} / \mathrm{sp}^{3}$ composition. This might suggest that the fluence interval used to fabricate the carbon layers was too short to appreciate remarkable changes on the photocurrent characteristics of the devices, or that the subsequent annealing of the sample ultimately controls the final conductivity. Either way, it suggests that the PLD method produces carbon electrodes with high repeatability.

The devices showed low leakage current, good stability and repeatability of the photocurrent under radiation, fast response and dose rate linearity which make them suitable for applications in medical dosimetry. It should be mentioned that in all cases it was possible to apply a bias voltage up to $\pm 50 \mathrm{~V}$ in order to keep stable the photocurrent under radiation.

As a further step, a new diamond detector based on a $500 \mu \mathrm{m} \mathrm{scCVD}$ diamond sample was developed with both electrodes fabricated at the laser fluence of $3.2 \mathrm{~J} \cdot \mathrm{cm}^{-2}$. Compared to the other results, the device showed very low gain and lack of linearity respect the dose rate. The former in particular could be related to the low voltage applied.

The common drawback observed in all the devices was the instability of the photocurrent recorded under irradiation which in turn reflected in a limitation of the maximum voltage applied. This could be related either to the poor adhesion of the carbon electrodes on the diamond surfaces or to the low thickness of the deposited carbon films. To confirm this, future experiments are being planned with the aim to check both the mechanical stability of the electrodes and the optimization of the deposition process. Moreover, further tests will be 
performed both in order to fabricate carbon electrodes in a broader range of laser fluences and to extend the device characterization over a wider range of X-ray energies and dose rates.

\section{References}

[1] B. Planskoy, Evaluation of diamond radiation dosemeters, Phys. Med. Biol. 25(3), 519 (1980).

[2] J. Bol, S. Müller, E. Berdermann, W. de Boer, A. Furgeri, M. Pomorski, C. Sander, Diamond thin film detectors for beam monitoring devices, Phys. Status Solidi A 204(9), 2997 (2007).

[3] C. J. H. Wort, R. S. Balmer, Diamond as an electronic material, Mater. Today 11(1-2), 22 (2007).

[4] M. A. E. Abdel-Rahman, A. Lohstroh, I. Jayawardena, S. J. Henley, The X-ray detection performance of polycrystalline CVD diamond with pulsed laser deposited carbon electrode, Diamond Relat. Mater. 22, 70 (2012).

[5] S. J. Henley, J. D. Carey, S. R. P. Silva, G. M. Fuge, M. N. R. Ashfold, D. Anglos, Dynamics of confined plumes during the short and ultra-short pulsed laser ablation of graphite, Phys. Rev. B. 72, 205413 (2005).

[6] Y. Miyajima, S. J. Henley, G. Adamopoulos, V. Stolojan, E. Garcia-Caurel, B. Drévillon, J. M. Shannon, S. R. P. Silva, Pulsed laser deposited tetrahedral amorphous carbon with high $\mathrm{sp}^{3}$ fractions and low optical bandgaps, J. Appl. Phys. 105, 073521 (2009).

[7] K. D. G. I. Jayawardena, Y. Y. Tan, J. Fryar, H. Shiozawa, S. R. P. Silva, S. J. Henley, G. M. Fuge, B. S. Truscott, M. N. R. Ashfold, Highly conductive nanoclustered carbon:nickel films grown by pulsed laser deposition, Carbon 49 (12), 3781 (2011).

[8] H. Kuzmany, R. Pfeiffer, N. Salk, B. Günther, The mystery of the $1140 \mathrm{~cm}-1$ Raman line in nanocrystalline diamond films, Carbon 42, 911 (2004).

[9] A. J. Whitehead, R. Airey, C. M. Buttar, J. Conway, G. Hill, S. Ramkumar, G. A. Scarsbrook, R. S. Sussmann S. Walker, CVD diamond for medical dosimetry applications, Nucl. Instrum. Methods Phys. Res. A, 460, 20 (2001).

[10] I.A.E. Agency, Calibration of dosimeters used in radiotherapy in Technical reports series, vol. 374 1994 Vienna.

[11] J. F. Fowler, in: F. H. Attix, W. C. Roesch (Eds.), Radiation. Dosimetry, Academic Press Inc, Chapter 14, 308 (1966).

[12] A. Fidanzio, L. Azario, P. Viola, P. Ascarelli, E. Cappelli, G. Conte, A. Piermattei, Photon and electron beam dosimetry with a CVD diamond detector, Nucl. Instrum. Methods Phys. Res. A 524(1-3), 115 (2004).

[13] M. Bucciolini, E. Borchi, M. Bruzzi, M.Casati, G.A.P. Cirrone, G. Cuttone, C. De Angelis, I. Lovik, S. Onori, L. Raffaele, S. Sciortino, Diamond dosimetry: Outcomes of the CANDIDO and CONRAD INFN projects, Nucl. Instrum. Methods Phys. Res. A 552(1-2), 189 (2005).

[14] C. Descamps, D. Tromson, N. Tranchant, A. Isambert, A. Bridier, C. De Angelis, S. Onori, M. Bucciolini, P. Bergonzo, Clinical studies of optimised single crystal and polycrystalline diamonds for radiotherapy dosimetry, Radiation Measurements, 43(2-6), 933 (2008).

[15] F. Schirru, K. Kisielewicz, T. Nowak, B. Marczewska, Single crystal diamond detector for radiotherapy, J. Phys. D 43(26), 1 (2010). 
[16] P. W. Hoban, M. Heydarian, W. A. Beckham, A. H. Beddoe, Dose rate dependence of a PTW diamond detector in the dosimetry of a 6 MV photon beam, Phys. Med. Biol. 39, 1219 (1994).

[17] M. A. E. Abdel-Rahman, A. Lohstroh, P. J. Sellin. The effect of annealing on the X-ray induced photocurrent characteristics of CVD diamond radiation detectors with different electrical contacts, Phys, Status Solidi A 208, 2079 (2011).

[18] S. Rankumar, C. M. Buttar, J. Conway, A. J. Whitehead, R. S. Sussmann, G. Hill, S. Walker, An assessment of radiotherapy dosimeters based on CVD grown diamond, Nucl. Instrum. Methods Phys. Res., A 460(2-3), 401 (2001). 